\begin{tabular}{l|l} 
REVISTA & $\begin{array}{l}\text { Revista Educación } \\
\text { ISSN: 0379-7082 } \\
\text { ISSN: 2215-2644 } \\
\text { revedu@ gmail.com } \\
\text { Universidad de Costa Rica } \\
\text { Costa Rica }\end{array}$
\end{tabular}

\title{
Evaluación de las funciones que desempeña el profesorado en educación superior tecnológica
}

Carranza, Arturo; Castro Ramírez, Sandra Lucía; García Domínguez, Mónica

Evaluación de las funciones que desempeña el profesorado en educación superior tecnológica

Revista Educación, vol. 45, núm. 2, 2021

Universidad de Costa Rica, Costa Rica

Disponible en: https://www.redalyc.org/articulo.oa?id=44066178037

DOl: https://doi.org/10.15517/revedu.v45i1.44372

\section{(c) (1) 90}

Esta obra está bajo una Licencia Creative Commons Atribución-NoComercial-SinDerivar 3.0 Internacional. 


\section{Evaluación de las funciones que desempeña el profesorado en educación superior tecnológica}

Evaluación de las funciones que desempeña el profesorado en educación superior tecnológica Teaching Faculty Assessment in Higher Technological Education

Arturo Carranza

Tecnológico Nacional de México, México

d_docencia01@tecnm.mx

iD https://orcid.org/0000-0002-9204-9826

Sandra Lucia Castro Ramirez

Tecnológico Nacional de México, México

d_docencia03@tecnm.mx

iD https://orcid.org/0000-0003-1628-5752

Mónica Garcia Dominguez

Tecnológico Nacional de México, México

d_docencia02@tecnm.mx

iD https://orcid.org/0000-0002-7004-2586
DOI: https://doi.org/10.15517/revedu.v45i1.44372

Redalyc: https://www.redalyc.org/articulo.oa? $\mathrm{id}=44066178037$

Recepción: 14 Noviembre 2020

Aprobación: 08 Enero 2021

\section{Resumen:}

Motivación: La evaluación del desempeño docente está asociada a la opinión del estudiantado y escasamente las instituciones integran cuestionarios que evalúen la perspectiva de la autoridad. Objetivo: Evaluar las funciones que desempeña el profesorado del Tecnológico Nacional de México (TecNM). Metodología: Se realizó una investigación no experimental de corte cuantitativo y diseño transversal, donde las dimensiones de las funciones que desempeña el profesorado y sus indicadores asociados fueron definidos por un grupo de personas docentes del TecNM; el instrumento resultante fue de 15 ítems en 4 dimensiones que incluyeron las funciones que desempeña el profesorado en docencia, tutoría, investigación y vinculación, esto desde la perspectiva de las jefaturas de departamento académico sobre una escala Likert de 5 puntos. Resultados: La prueba del instrumento con 5,781 personas exhibió muy buenos índices de grado, de acuerdo con las personas jueces — Lawshe, V de Aiken y Osterlind- y con la confiabilidad alfa de Cronbach y omega de McDonald para las cuatro dimensiones. Esto implicó un riguroso proceso de revisión por un jurado experto y una alta consistencia interna del instrumento. Los resultados del análisis factorial exploratorio indicaron que no hubo factores irrelevantes del constructo, los ítems incluidos, considerados como fuertes, coincidieron con la dimensión asignada teóricamente. Los resultados del análisis factorial confirmatorio verificaron el ajuste global del modelo propuesto; todos los ítems tuvieron contribuciones significativas en sus dimensiones. Conclusiones: La utilización del instrumento tiene potencial para la medición del nivel de efectividad de las funciones que desempeña el profesorado de educación superior tecnológica.

Palabras clave: Evaluación docente, Competencias docentes, Análisis factorial, Cuestionario, Educación superior, Tecnología.

\section{Abstract:}

Teacher assessments tend to be associated with the opinion of the student body and higher learning institutions rarely use questionnaires to assess the perspective of school authorities. This study uses a non-experimental, quantitative and cross-sectional approach to design a questionnaire that evaluates the faculty of the National Institute of Technology of Mexico (TecNM). Associated indicators were defined by a group of TecNM instructors. The resulting questionnaire consists of 15 questions in 4 areas that assesses duties and tasks performed by the teaching faculty as well as other instructor activities such as, tutoring, research and liaison, based on the perspective of the academic department and using a 5-point Likert scale. The instrument was answered by 5,781 people and received good index reviews by the judges, based on Lawshe, V of Aiken and Osterlind- with Cronbach's alpha and $\mathrm{McD}$ onald's omega reliability for the survey's four question areas. High scores were attained through a rigorous review process 
executed by an expert jury as well as an internally consistent instrument. The results of the exploratory factor analysis indicate that there were no irrelevant factors in the questionnaire design. Included items were deemed to be strong and coincided with the theoretically assigned dimension. The results of the confirmatory factor analysis verified the overall fit of the proposed model; all items proved to offer significant contributions for each of their fields. It was concluded that this survey does, in fact, have the potential to gauge the effectiveness of how well the teaching staff fulfills its duties at this higher education institution.

KEYWORDS: Teacher Assessment, Teacher Qualifications, Factor Analysis, Questionnaire, Higher Education, Technology.

\section{INTRODUCCIÓN}

Las instituciones de educación superior forman y desarrollan en el estudiantado competencias que le permita ser agente de cambio en su campo profesional. Por lo que la calidad, competitividad y actualización de los conocimientos del profesorado, es el factor más determinante en el aprendizaje y rendimiento del estudiantado (Sanders, Wright y Horn, 1997). En este contexto, la evaluación del desempeño docente es importante para elevar la calidad del proceso de enseñanza-aprendizaje, fortalecer la formación profesional del profesorado y alcanzar los objetivos organizacionales.

Los sistemas de evaluación utilizan varios instrumentos para valorar de forma holística las tareas del profesorado en el aula, las actividades con el estudiantado, el servicio que brinda a la sociedad en la solución de problemas y su dedicación para el desarrollo continuo de sus competencias (Pacheco, Ibarra, Iñiguez, Lee y Victoria, 2018). Cada institución establece las características, conocimientos y habilidades que el profesorado tiene para la enseñanza-aprendizaje, la formación integral del estudiantado, el desarrollo profesional, la ética del profesorado, la gestión del aprendizaje y la gestión institucional (Perrenoud, 2004; Zabalza, 2007).

Lo anterior implica que las instituciones deben definir instrumentos para la evaluación del desempeño del personal docente y mecanismos de participación para la comunidad administrativa, directiva, docente y estudiantil en este proceso. La forma en la que se lleva a cabo esta evaluación es mediante la opinión del estudiantado, cuestionarios de autoridades, la autoevaluación del profesorado y observación por pares académicos (Rueda, Luna, García y Loredo, 2010; Moreno, Nava y Campos, 2014).

Finalmente, de acuerdo con Cancino y Márquez (2015), "los sistemas de evaluación de desempeño son absolutamente necesarios en toda institución para mejorar sus procesos y alcanzar óptimos estándares de calidad" (p. 36), por lo que, para lograr los objetivos estratégicos, las instituciones deben diseñar sus sistemas de evaluación a medida, a través del análisis de su contexto y condiciones internas.

Con base en las variables mencionadas en los párrafos anteriores, en este artículo se planteó el objetivo de evaluar las funciones que desempeña el profesorado del Tecnológico Nacional de México. Para ello, se diseñó y validó un instrumento que mide el grado de desempeño de las actividades que realiza el profesorado en las dimensiones de: docencia, tutoría, investigación y vinculación, estas desde la perspectiva de las jefaturas de departamento académico.

El contenido se encuentra organizado como sigue: la segunda sección presenta la revisión de la literatura; la tercera sección describe la recolección de los datos, construcción y confiabilidad del instrumento, así como las pruebas de validez realizadas; la cuarta sección muestra los resultados obtenidos; las discusiones y conclusiones se mencionan en la quinta y sexta sección, respectivamente; y los anexos muestran las tablas del instrumento, población participante y resultados.

\section{MARCo TEÓRICo}

Una educación de calidad tiene tres componentes: el aprendizaje real de los programas de estudio logrado por el estudiantado, las condiciones suficientes que necesitan los individuos e individuas para desarrollarse como persona e integrarse a la sociedad, y los procesos de servicios que brinda la institución al estudiantado (Toranzos,1996). Por lo que la práctica de la evaluación del desempeño docente es un buen ejercicio 
sistemático para medir la calidad del servicio educativo, esto con el uso de indicadores del estado que guarda el proceso en un determinado tiempo, para con esta información determinar mecanismos de mejora (Tejedor, 2003).

La evaluación del desempeño docente es una tarea compleja que tiene como objetivo fortalecer a las instituciones en la calidad del servicio de educación que ofrece a la sociedad (Maussa, 2014; Pacheco et al., 2018). La práctica de la evaluación generalmente está orientada a dos propósitos: formativo, para el desarrollo profesional, o sumativo, para la rendición de cuentas (Tejedor y Jornet, 2008; Montoya, Arbesú, Contreras y Conzuelo, 2014; Gómez y Valdés, 2019). De acuerdo con Isoré (2009), la evaluación con propósito formativo, busca conocer la práctica real del profesorado y es utilizada para la mejora de sus competencias, mientras que el sumativo trata de medir la calidad del rendimiento del profesorado y es útil para la toma de decisiones en su contratación, promoción y titularidad.

La evaluación del desempeño docente implica definir, en un contexto institucional, las dimensiones que caractericen un perfil de lo que se espera del profesorado. Aunque, es difícil establecer en todas las instituciones de educación superior un marco de referencia de competencias docentes, la experiencia acumulada mediante la literatura indica que se han incluido competencias relacionadas con las funciones de: docencia, investigación, tutoría, gestión y administración (Comellas, 2002; Tejedor, 2003; Perrenoud, 2004; Zabalza, 2007; Torra et al., 2012; López, González y de León, 2014). Por otro lado, si se pretende hacer realidad la escuela para todas las personas, entonces, sería deseable que el perfil del profesorado considere los aspectos de la inclusión y lo socioemocional. El primero conlleva a las competencias docentes en relación a la diversidad de las personas, al reconocer sus diferencias y necesidades educativas específicas (Fernández, 2013; Ayala y O'Hinggins, 2019), mientras que el segundo precisa que los aspectos socioafectivos y emocionales del profesorado impactan positivamente cuando los tienen, y negativamente cuando carecen de ellos (Arreola, 2019).

De acuerdo con Cruz-Aguayo, Hincapié y Rodríguez (2020), elementos centrales en los procedimientos de evaluación del desempeño docente han sido los instrumentos, debido a que los resultados otorgados por estos permiten identificar al profesorado que tiene un alto impacto en el estudiantado (información válida), así como un reflejo típico del desempeño del profesorado dentro de la institución (información confiable). Rueda et al. (2010) y Moreno et al. (2014) exponen que el procedimiento más estudiado y empleado por las instituciones de educación superior en la evaluación del desempeño docente es el cuestionario de opinión del estudiantado, mientras que otros procedimientos utilizados en menor medida han sido la opinión del personal directivo y la autoevaluación del profesorado.

En la literatura consultada se encontraron varios estudios de análisis factoriales de la opinión del estudiantado para medir la efectividad del profesorado (Zambrano, Meda y Lara, 2005; Pimienta, 2014; Moreno et al., 2014; Luna y Reyes, 2015; Dios, Calmaestra y Rodríguez-Hidalgo, 2018); los ítems de estos estudios se pueden agrupar en cinco dimensiones que caracterizan la actuación del profesorado: dominio de la asignatura, métodos y materiales de enseñanza, relación con el estudiantado, entusiasmo por la docencia y la forma de evaluación. En menor medida, se encontraron otros estudios desde la perspectiva del profesorado (Hoi, Zhou, Teo y Nie, 2017; Ruiz-Corbella y Aguilar-Feijoo, 2017; Aguiar, Gutiérrez y Gutiérrez, 2018) y otro desde la perspectiva de la autoridad de la institución (Mohamadi y Malekshahi, 2018).

México no es un país que esté alejado de lo expuesto hasta este punto, desde 1920, algunas instituciones de educación superior comenzaron a solicitar al estudiantado que evaluara el desempeño del profesorado (Gómez y Valdés, 2019); sin embargo, de acuerdo con Rueda et al. (2010), a partir de 1990 la evaluación sufrió una transformación considerable por lineamientos gubernamentales, los cuales estaban orientados al rendimiento del profesorado y asociados a estímulos de índole económico. Así, diversas instituciones de educación superior han definido sus criterios de evaluación y utilizado las encuestas de opinión del estudiantado para valorar el rendimiento del profesorado. 
En este sentido, el TecNM es una institución de educación superior tecnológica conformada por 254 campus distribuidos en los 32 estados de México, que ha llevado a cabo procesos de evaluación de su profesorado en forma gradual, principalmente en dos sentidos: el primero es en el ingreso y promoción de categoría que, desde 1982, la institución ha implementado mediante exámenes de oposición aplicados por una comisión de personas docentes (Secretaría de Educación Pública [SEP], 1982); el segundo es el desempeño docente que, a partir de 1992, se ha medido a través de cuestionarios de opinión del estudiantado, los cuales han sido orientados al proceso de enseñanza-aprendizaje en el aula (Dirección General de Educación Superior Tecnológica [DGEST], 2006). Si bien es cierto, estas dos estrategias institucionales han fortalecido la cultura de evaluación del profesorado en el TecNM y han sido utilizadas para la toma de decisiones administrativas, también se observa que no aparecen modelos de evaluación que permitan proporcionar información desde otros actores y donde se utilicen otros instrumentos, tales como informes de la autoridad, observación en el aula, pruebas estandarizadas al profesorado y estudiantado, portafolio del profesorado, y autoevaluación del profesorado. Esta situación institucional, y la escasa literatura de estudios orientados a la evaluación de cuestionarios respondidos por la autoridad, justifica la necesidad de conocer la percepción de las jefaturas de departamento académico sobre la valoración de las funciones que desempeña el profesorado en el TecNM.

\section{MÉtodo}

Esta investigación tiene como contexto sociodemográfico la población del TecNM y es no experimental cuantitativa con diseño transversal descriptivo.

\subsection{Consideraciones éticas}

La investigación se realizó con previa autorización del TecNM, los datos recolectados de las personas participantes fueron usados para fines estadísticos y de investigación, se ha respetado la confidencialidad de las personas participantes y se ha cumplido con las consideraciones éticas del TecNM, por lo que en este trabajo no se mencionan datos de personas.

\subsection{Procedimiento}

El desarrollo y ensayo del instrumento se llevó a cabo en seis fases. En la fase I, se revisó la literatura relacionada con la teoría de la evaluación docente (informada previamente). En la fase II, se elaboró la versión inicial del instrumento de evaluación de las funciones que desempeña el profesorado del TecNM (agosto-septiembre de 2019). En la fase III, se realizó la validación de contenido por el jurado experto (octubre de 2019). En la fase IV, se estimó la validez de comprensión mediante la estadística descriptiva y el análisis de ítems (noviembre de 2019). En la fase V, se examinó la validez del constructo mediante el análisis factorial exploratorio y confirmatorio - AFE y AFC - (enero-febrero 2020). Finalmente, en la fase VI, se determinó la consistencia interna con el cálculo de los coeficientes alfa de Cronbach $(\alpha)$ y omega de McDonald $(\omega)$ de la fase V.

\subsection{Participantes}

Un total de 5781 personas del TecNM participaron en este estudio, el $39.5 \%$ fueron mujeres y el $60.5 \%$ hombres. En la Tabla 1 se resumen las características de edad, sexo, nivel de estudios, tipo de personal y horas de contratación de las personas participantes, $N_{1}=9$ en el método de evaluación por un jurado experto, $N$ 
${ }_{2}=500$ en la prueba piloto, $N_{3}=2636$ en el AFE y $N_{4}=2636$ en el AFC. La selección fue de manera no probabilística para $N_{1}$ y probabilística para $N_{2}, N_{3}$ y $N_{4}$, en términos globales se observa que la edad con mayor participación se encuentra en el rango de 40 y 50 años, el nivel de estudios que sobresale es el de maestría y la mayoría ostenta una plaza de tiempo completo.

TABLA 1

Datos descriptivos de las personas participantes en el estudio

\begin{tabular}{|c|c|c|c|c|c|}
\hline \multirow{2}{*}{ Características } & & Jurado $N_{1}=9$ & Piloto $\mathrm{N}_{2}=500$ & $\mathrm{AFE} N_{3}=2636$ & $\mathrm{AFC} N_{4}=2636$ \\
\hline & & núm. (\%) & núm. (\%) & núm. (\%) & núm. (\%) \\
\hline \multirow{12}{*}{ Edad } & {$[24-30]$} & $0(0)$ & $16(3.2)$ & $110(4.2)$ & $101(3.8)$ \\
\hline & $(30-40]$ & $4(44.4)$ & $128(25.6)$ & $736(27.9)$ & $742(28.2)$ \\
\hline & $(40-50]$ & $4(44.4)$ & $178(35.6)$ & $816(31.0)$ & $813(30.8)$ \\
\hline & $(50-60]$ & 1(11.2) & $114(22.8)$ & $643(24.4)$ & $625(23.7)$ \\
\hline & $(60-70]$ & $0(0)$ & $56(11.2)$ & $292(11.1)$ & $311(11.8)$ \\
\hline & $(70-83]$ & $0(0)$ & $8(1.6)$ & $39(1.5)$ & $44(1.7)$ \\
\hline & & & & & \\
\hline & $\begin{array}{l}\text { Desviacion } \\
\text { estándar }\end{array}$ & 4.27 & 10.42 & 10.74 & 10.90 \\
\hline & Media & 43 & 47.12 & 46.91 & 47.21 \\
\hline & Mediana & 42 & 46 & 46 & 46 \\
\hline & Asimetría & 1.20 & 0.28 & 0.29 & 0.31 \\
\hline & Curtosis & 1.33 & -0.58 & -0.61 & -0.61 \\
\hline \multirow{2}{*}{ Sexo } & Hombre & $5(55.6)$ & $326(65.2)$ & $1606(60.9)$ & $1561(59.2)$ \\
\hline & Mujer & $4(44.4)$ & $174(34.8)$ & $1030(39.1)$ & $1075(40.8)$ \\
\hline \multirow{4}{*}{ Nivel de Estudios } & Licenciatura & $O(0)$ & $145(29.0)$ & $788(29.9)$ & $740(28.1)$ \\
\hline & Especialidad & $0(0)$ & $7(1.4)$ & $15(0.6)$ & $13(0.5)$ \\
\hline & Maestría & $7(77.8)$ & $252(50.4)$ & $1269(48.1)$ & $1380(52.4)$ \\
\hline & Doctorado & $2(22.2)$ & $96(19.2)$ & $564(21.4)$ & $503(19.1)$ \\
\hline \multirow{2}{*}{ Tipo de Personal } & Profesorado & $9(100)$ & $418(83.6)$ & $2192(83.2)$ & $2195(83.3)$ \\
\hline & Directivo & $0(0)$ & $82(16.4)$ & $444(16.8)$ & $441(16.7)$ \\
\hline \multirow{4}{*}{ Horas de contratación } & Asignatura & $0(0)$ & $80(16.0)$ & $510(19.3)$ & $476(18.1)$ \\
\hline & $3 / 4$ tiempo & $0(0)$ & $37(7.4)$ & $153(5.8)$ & $173(6.6)$ \\
\hline & $1 / 2$ tiempo & $O(0)$ & $20(4.0)$ & $83(3.1)$ & $88(3.3)$ \\
\hline & Completo & $9(100)$ & $363(72.6)$ & $1890(71.7)$ & $1899(72)$ \\
\hline
\end{tabular}

Fuente: Elaboración propia.

Nota: AFE = análisis factorial exploratorio y AFC = análisis factorial confirmatorio.

En el caso de las personas que forman parte del jurado experto, estas cumplieron con cuatro criterios: experiencia en el diseño de instrumentos, experiencia en la docencia, experiencia directiva y publicaciones. El $100 \%$ tiene experiencia en la elaboración de instrumentos; el $77.8 \%$ tiene publicaciones en el área de educación, el $44.4 \%$ en tecnología y el $33.3 \%$ en ingeniería; el tiempo de experiencia en la docencia fue de un rango de 11-27 años, con un valor medio de $17 \pm 5.8$ años; por último, el tiempo de experiencia directiva se encontró en el rango de 1-13 años con un valor medio de $5 \pm 4.7$ años

\subsection{Instrumento}

Con base en la revisión de la literatura y las actividades que realiza el profesorado del TecNM, se elaboró, con un grupo de expertos, un constructo para evaluar las funciones que desempeña el profesorado desde la percepción de la autoridad (ver Tabla 2); se delimitan cuatro dimensiones: docencia, tutoría, investigación y vinculación. Estas dimensiones están alineadas con las tareas señaladas en el reglamento del personal docente de la Secretaría de Educación Pública (SEP, 1982): horas dedicadas para clases, elaboración de programas de estudio y prácticas, materiales didácticos, asesorías, comisiones, investigación, procesos de titulación, desarrollo de proyectos, cargos administrativos, tutoría y desarrollo personal o profesional. 
TABLA 2

Dimensiones, indicadores e ítems del instrumento final para el profesorado

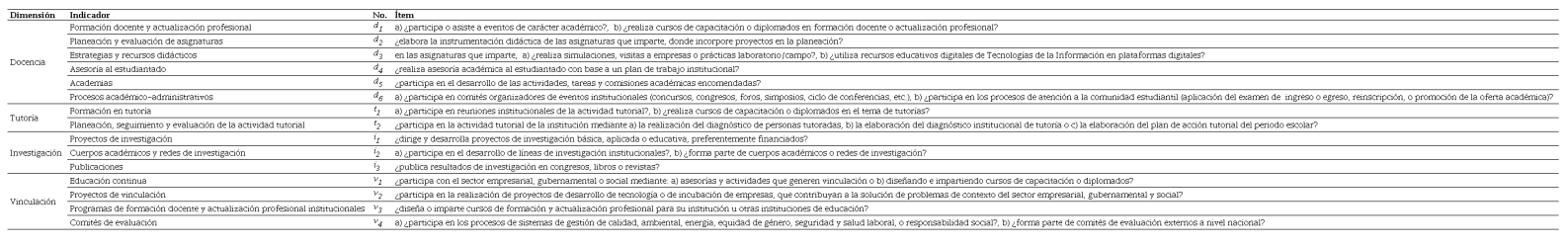

Fuente: Elaboración propia.

Se elaboraron 15 ítems de escala tipo Likert de 5 puntos con niveles de desempeño representados por: 1 $=$ no suficiente, 2 suficiente, $3=$ bien, $4=$ muy bien y $5=$ excelente, los cuales se encuentran distribuidos en las dimensiones de la siguiente manera:

a) Docencia $\left(d_{1} a d_{6}\right)$. Actividades del profesorado que logran transformar las competencias del perfil de egreso y los programas de asignaturas en conocimiento y práctica para el estudiantado.

b) Tutoría $\left(t_{1} y t_{2}\right)$. Actividades del profesorado de acompañamiento y conducción del estudiantado en el desarrollo y éxito de su trayectoria académica.

c) Investigación $\left(\begin{array}{lll}i_{1} & a & i_{3}\end{array}\right)$. Actividades del profesorado que promueven la generación, transferencia y divulgación del conocimiento científico-tecnológico relacionado con su área de formación profesional o línea de especialización, estas actividades favorecen la integración del estudiantado en investigación y desarrollo tecnológico.

d) Vinculación $\left(v_{1} a v_{4}\right)$. Actividades del profesorado que consolidan agentes de cambio en la sociedad para dar respuesta a las necesidades económicas y sociales del entorno, y fortalecen la formación integral del estudiantado.

En la dimensión de docencia, los ítems $d_{2} d_{3}, d_{4}$ y $d_{5}$, probablemente son los que coincidan con lo planteado en diversos estudios (Tejedor, 2003; Perrenoud, 2004; Zabalza, 2007; López et al., 2014), que hacen referencia a que el perfil del profesorado incluye la planificación de asignaturas, selección de contenidos, bibliografía, material de apoyo didáctico, diseño de escenarios de aprendizaje, interacción con el estudiantado y trabajo colegiado. El ítem $d_{l}$ reconoce la acreditación de cursos y la asistencia a eventos académicos externos a la institución, esto responde a que las necesidades de formación y actualización de las competencias disciplinares son subsanadas por el propio profesorado o por las academias del departamento académico (Torra et al., 2012). El ítem $d$. identifica la participación del profesorado en la institución, ya sea en comités de eventos académicos o procesos administrativos dirigidos al estudiantado como inscripción, examen de ingreso y promoción de la oferta educativa.

En la dimensión de tutoría, los ítems $t_{1}$ y $t_{2}$ reconocen la participación del profesorado en reuniones colegiadas de la acción tutorial, su capacitación en el tema y el diagnóstico del tutorado (o institucional). Esto está acorde con lo que plantea Comellas (2002): en primer término, que la formación y actualización del profesorado que realiza la acción tutorial es imprescindible en una institución; en segundo término, esta acción tutorial debe valorar, en estudiantado y sus familias, los aspectos personales y sociales en el contexto de la institución. 
En la dimensión de investigación, los ítems $i_{1}$ e $i_{3}$, de acuerdo con Montoya et al. (2014), son fundamentales, debido a que identifican la contribución que realiza el profesorado a través del desarrollo de proyectos científico-tecnológicos y la publicación de sus hallazgos en revistas. En esta misma línea de ideas, el ítem $i_{2}$ reconoce la partición y liderazgo del profesorado en grupos y cuerpos académicos de una determinada línea de investigación dentro y fuera de la institución.

En la dimensión de vinculación, los ítems $v_{1}$ y $v_{2}$ reconocen las actividades emprendidas de capacitación y el desarrollo de proyectos en el mundo laboral, para dar respuesta a las necesidades del entorno (RuizCorbella y Aguilar-Feijoo, 2017). El ítem $v$. identifica al profesorado que transmite su experiencia docente y profesional al personal docente de otra institución, ya sea al instruir o diseñar materiales de cursos de capacitación. El ítem $v_{4}$ reconoce la participación del profesorado miembro o auditor líder en los grupos de sistemas de gestión (ambiental, laboral, equidad, higiene, energía) y comités relacionados con ciencia, tecnología e innovación (organismos acreditadores, comisiones estatales para la planeación de la educación, fondos de emprendedores, entre otros).

Para obtener los 15 ítems descritos anteriormente, la versión inicial fue sometida a dos procesos de evaluación: validación de contenido y de comprensión.

Validez de contenido. Se utilizó el método de evaluación por un jurado experto, donde $N_{I}$ realizó la evaluación de los 21 ítems de la versión inicial del instrumento (fase III). Se utilizaron tres criterios de eliminación de ítem, el primero fue el coeficiente . de Aiken, que a través de una escala Likert de 5 puntos midió el grado acuerdo de la claridad del lenguaje, pertinencia práctica y relevancia teórica; el segundo fue el índice de validez de contenido $(I V C)$ de Lawshe, que mediante una escala Likert de 3 puntos midió el grado de acuerdo si el ítem es esencial, útil o innecesario; el tercero fue el índice de congruencia de Osterlind, que midió por medio de una escala Likert de 3 puntos el grado de consenso sobre la ubicación de cada ítem en la dimensión teórica.

Validez de comprensión. En la prueba piloto (fase IV), $N_{2}$ analizó el grado de comprensión de los enunciados de los ítems y los parámetros estadísticos asociados a la media, desviación estándar, asimetría, curtosis, grados de dificultad, discriminación y fiabilidad, los cuales determinaron la conservación de ítems.

\subsection{Validez de constructo}

Durante la fase V, el AFE encontró un modelo de ítems en factores y el AFC verificó su estructura factorial y probó el ajuste de datos del modelo.

AFE. Con $N$. se comprobó la viabilidad de realizar el AFE calculando el determinante de la matriz de correlaciones, el índice de adecuación muestral Kaiser-Meyer-Olkin (KMO) y la prueba de esfericidad de Bartlett. El número de factores se determinó con el análisis en paralelo de los valores propios mayores a los que se obtendrían de manera aleatoria, la extracción se realizó con el análisis de componentes principales, y la rotación utilizó el método de oblicuo oblimin.

AFC. Con $N_{4}$ se realizó el AFC, el método de máxima verosimilitud. La bondad de ajuste se determinó con la evaluación del valor de chi cuadrado $\left(c^{2}\right)$ y sus grados de libertad $(g l)$ asociados, el índice de bondad de ajuste $(G F I)$, el índice ajustado de bondad de ajuste $(A G F I)$, el índice de ajuste normado $(N F I)$, el índice TuckerLewis $(T L I)$, el índice de ajuste comparativo $(C F I)$, el error medio cuadrático de aproximación $(R M S E A)$ y la media cuadrática residual estandarizada $(S R M R)$.

3.6 Análisis de confiabilidad

La confiabilidad del constructo (fase VI) se determinó calculando los coeficientes $\alpha$ y $\omega$ en el AFE y AFC. 


\section{Resultados}

Se utilizó el programa RStudio para el análisis estadístico, y la plataforma Office365 y la nube de Azure del TecNM para la aplicación del instrumento. El primer resultado determina la versión final de un instrumento que valora las funciones que desempeña el profesorado, según las evidencias de validez y confiabilidad del constructo aplicado, y finalmente, la radiografía del desempeño del profesorado.

\subsection{Validez de contenido y validez de comprensión}

La versión preliminar del instrumento, elaborada por un grupo colegiado del TecNM, estuvo formada por 21 ítems que atienden los procesos académico-administrativos, investigativos y de vinculación del profesorado. Los ítems se asociaron a 4 dimensiones: docencia (7), tutoría (4), investigación (4) y vinculación (6).

Validez de contenido. La revisión rigurosa del jurado experto eliminó el $28.57 \%$ de los ítems, a saber, formación académica $\left(d_{7}\right)$, inducción de nuevo ingreso $\left(t_{3}\right)$, desempeño tutorial $\left(t_{4}\right)$, asesoría en proyectos de investigación $\left(i_{4}\right)$, asesoría de residencia profesional $\left(v_{5}\right)$ y actividades de vinculación $\left(v_{6}\right)$; estos fueron eliminados por no ser relevantes y representativos, debido a su $I C V £ 0.56$ y $V$ de Aiken $£ 0.5$ (ver Tabla 3 ).

TABLA 3

IVC de Lawshe y coeficiente $\mathrm{V}$ de Aiken de la versión inicial del instrumento

\begin{tabular}{|c|c|c|c|c|c|c|c|c|c|}
\hline \multirow[t]{2}{*}{ Dimensión } & \multirow[t]{2}{*}{ Ítem } & \multirow{2}{*}{$\begin{array}{l}\text { Claridad del } \\
\text { lenguaje }\end{array}$} & \multirow[b]{2}{*}{$\mathrm{V}$} & \multicolumn{2}{|c|}{$\begin{array}{l}\text { Pertinencia } \\
\text { práctica }\end{array}$} & \multicolumn{2}{|c|}{$\begin{array}{l}\text { Relevancia } \\
\text { teórica }\end{array}$} & \multirow[t]{2}{*}{$V$ total } & \multirow[t]{2}{*}{ ICV } \\
\hline & & & & $\mathrm{ME} \pm \mathrm{DE}$ & $\mathrm{V}$ & $\mathrm{ME} \pm \mathrm{DE}$ & $\mathrm{V}$ & & \\
\hline \multirow{7}{*}{ Docencia } & $d_{1}$ & $4.8 \pm 0.6$ & 0.94 & $4.2 \pm 0.6$ & 0.81 & $4.7 \pm 0.5$ & 0.92 & 0.89 & 1.00 \\
\hline & $a_{2}$ & $4.2 \pm 0.9$ & 0.81 & $4.1 \pm 0.7$ & 0.78 & $4.4 \pm 0.7$ & 0.86 & 0.81 & 0.78 \\
\hline & $d_{3}$ & $3.6 \pm 1.3$ & 0.64 & $4.3 \pm 0.7$ & 0.83 & $3.8 \pm 1.0$ & 0.69 & 0.72 & 0.78 \\
\hline & $a_{4}$ & $4.8 \pm 0.6$ & 0.94 & $4.2 \pm 1.0$ & 0.81 & $4.7 \pm 0.7$ & 0.92 & 0.89 & 0.78 \\
\hline & $d_{5}$ & $4.7 \pm 0.5$ & 0.92 & $4.9 \pm 0.3$ & 0.97 & $4.9 \pm 0.3$ & 0.97 & 0.95 & 0.78 \\
\hline & $d_{6}$ & $4.8 \pm 0.4$ & 0.94 & $4.6 \pm 0.7$ & 0.89 & $4.7 \pm 0.7$ & 0.92 & 0.92 & 1.00 \\
\hline & $d_{7}^{*}$ & $4.8 \pm 0.4$ & 0.94 & $1.4 \pm 0.8$ & 0.11 & $1.6 \pm 0.8$ & 0.14 & 0.40 & 0.33 \\
\hline \multirow{4}{*}{ Tutoria } & $t_{1}$ & $4.3 \pm 1.1$ & 0.83 & $4.3 \pm 0.7$ & 0.83 & $4.6 \pm 0.7$ & 0.89 & 0.85 & 1.00 \\
\hline & $t_{2}$ & $4.6 \pm 0.7$ & 0.89 & $4.2 \pm 0.8$ & 0.81 & $4.4 \pm 0.7$ & 0.86 & 0.85 & 1.00 \\
\hline & $t_{3}{ }^{*}$ & $4.6 \pm 0.5$ & 0.89 & $1.9 \pm 0.7$ & 0.22 & $2.3 \pm 0.8$ & 0.33 & 0.48 & 0.56 \\
\hline & $t_{4}^{*}$ & $3.7 \pm 0.9$ & 0.67 & $2.6 \pm 1.1$ & 0.39 & $2.8 \pm 0.8$ & 0.44 & 0.50 & 0.56 \\
\hline \multirow{4}{*}{ Investigación } & $i_{1}$ & $4.4 \pm 0.7$ & 0.86 & $4.2 \pm 0.6$ & 0.81 & $4.4 \pm 0.7$ & 0.86 & 0.84 & 1.00 \\
\hline & $i_{2}$ & $4.3 \pm 0.8$ & 0.83 & $4.4 \pm 0.7$ & 0.86 & $4.7 \pm 0.7$ & 0.92 & 0.87 & 1.00 \\
\hline & $i_{3}$ & $4.7 \pm 0.7$ & 0.92 & $4.7 \pm 0.5$ & 0.92 & $4.9 \pm 0.3$ & 0.97 & 0.94 & 0.78 \\
\hline & $i_{4}^{*}$ & $3.1 \pm 0.6$ & 0.53 & $1.3 \pm 0.7$ & 0.08 & $1.3 \pm 0.5$ & 0.08 & 0.23 & 0.56 \\
\hline \multirow{6}{*}{ Vinculación } & $v_{1}$ & $4.7 \pm 0.5$ & 0.92 & $4.6 \pm 0.5$ & 0.89 & $4.7 \pm 0.5$ & 0.92 & 0.91 & 1.00 \\
\hline & $v_{2}$ & $4.2 \pm 0.9$ & 0.81 & $4.3 \pm 0.7$ & 0.83 & $4.7 \pm 0.5$ & 0.92 & 0.85 & 1.00 \\
\hline & $v_{3}$ & $4.9 \pm 0.3$ & 0.97 & $4.4 \pm 0.8$ & 0.86 & $4.7 \pm 0.7$ & 0.92 & 0.92 & 0.78 \\
\hline & $v_{4}$ & $4.6 \pm 0.7$ & 0.89 & $4.6 \pm 0.5$ & 0.89 & $4.2 \pm 1.0$ & 0.81 & 0.86 & 0.78 \\
\hline & $\nu_{5}^{*}$ & $3.4 \pm 0.5$ & 0.61 & $1.7 \pm 0.7$ & 0.17 & $2.1 \pm 0.3$ & 0.28 & 0.35 & 0.56 \\
\hline & $\nu_{6}^{*}$ & $3.0 \pm 0.8$ & 0.50 & $2.0 \pm 0.7$ & 0.25 & $2.8 \pm 0.6$ & 0.44 & 0.40 & 0.56 \\
\hline Total & & $4.3 \pm 0.7$ & 0.82 & $3.7 \pm 0.7$ & 0.67 & $3.9 \pm 0.6$ & 0.72 & 0.74 & 0.79 \\
\hline
\end{tabular}


El jurado experto coincidió que $d_{7}$ se incluyera como experiencia adquirida en otros ítems, mientras que en los otros cinco ítems eliminados argumentaron que eran redundantes, porque estaban incluidos en otros ítems de la misma dimensión. La evaluación del jurado experto no modificó la relación ítem-dimensión ni eliminó ítems de la segunda versión $\left(d_{1}\right.$ a $d_{6}, t_{1}$ a $t_{2}, i_{1}$ a $i_{3}$, y $v_{1}$ a $\left.v_{4}\right)$, debido que los índices de Osterlind cumplieron con los criterios de inclusión en la dimensión (ver Tabla 4).

TABLA 4

Índices de congruencia de Osterlind de la segunda versión del instrumento

\begin{tabular}{llll}
\hline Criterio & Indicadores & \\
\cline { 2 - 4 } & $d_{1}, d_{6}, t_{2}, i_{2}, i_{3}$ & $d_{5}, t_{1}, i_{1}, v_{1}, v_{2}, v_{4}$ & $d_{2}, d_{3}, d_{4}, v_{3}$ \\
\hline Incluido [.6-1] & 1.00 & 0.78 & \\
\hline $\begin{array}{l}\text { Incluido, cerca de eliminarse } \\
{[.5-.6)}\end{array}$ & & 0.56 \\
\hline
\end{tabular}

Fuente: Elaboración propia.

Fuente: Elaboración propia.

Validez de comprensión. Los resultados de la prueba piloto no eliminaron ítems de la tercera versión del instrumento y se consideraron buenos ítems, debido a que el análisis de ítems de la Tabla 5 mostró que la asimetría y curtosis de los ítems fue aceptable por estar en el rango \pm 2 . El grado de dificultad de todos los ítems fue aceptable ${ }^{3} 0.2, £ 0.9$; su valor de discriminación $>0.4$ fue bueno, y su fiabilidad fue aceptable $>$ 0.8. En ambos procesos se realizaron correcciones de redacción y ortografía al instrumento. La versión final del instrumento se muestra en la Tabla 2.

TABLA 5

Análisis de los ítems de la tercera versión del instrumento

\begin{tabular}{|c|c|c|c|c|c|c|c|}
\hline \multirow{2}{*}{ Ítem } & \multicolumn{4}{|c|}{ Estadísticos descriptivos } & \multicolumn{3}{|l|}{ Ítem } \\
\hline & $\mathrm{ME}$ & $\mathrm{DE}$ & Asimetría & Curtosis & Dificultad & Discriminación & $\alpha$ si se elimina \\
\hline $\overrightarrow{d_{1}}$ & 2.90 & 1.51 & -0.07 & -1.11 & 0.58 & 0.609 & 0.898 \\
\hline$a_{2}$ & 3.82 & 1.19 & -0.69 & -0.24 & 0.76 & 0.550 & 0.900 \\
\hline$a_{3}$ & 4.06 & 1.13 & -1.27 & 0.98 & 0.81 & 0.598 & 0.899 \\
\hline$a_{4}$ & 3.84 & 1.30 & -1.16 & 0.70 & 0.77 & 0.644 & 0.897 \\
\hline$d_{5}$ & 4.36 & 1.17 & -1.93 & 3.07 & 0.87 & 0.550 & 0.900 \\
\hline$a_{6}$ & 3.81 & 1.31 & -0.95 & -0.01 & 0.76 & 0.566 & 0.899 \\
\hline$t_{1}$ & 2.66 & 1.89 & -0.14 & -1.43 & 0.53 & 0.595 & 0.899 \\
\hline$t_{2}$ & 3.41 & 1.79 & -0.91 & -0.58 & 0.68 & 0.603 & 0.898 \\
\hline$i_{1}$ & 2.30 & 1.80 & 0.06 & -1.37 & 0.46 & 0.682 & 0.895 \\
\hline$i_{2}$ & 1.90 & 1.57 & 0.18 & -1.23 & 0.38 & 0.688 & 0.895 \\
\hline$i_{3}$ & 2.00 & 2.05 & 0.33 & -1.59 & 0.40 & 0.595 & 0.899 \\
\hline$v_{1}$ & 2.04 & 1.68 & 0.04 & -1.36 & 0.41 & 0.680 & 0.895 \\
\hline$\nu_{2}$ & 1.84 & 1.68 & 0.27 & -1.35 & 0.37 & 0.646 & 0.896 \\
\hline$v_{3}$ & 1.32 & 1.71 & 0.93 & -0.65 & 0.26 & 0.493 & 0.902 \\
\hline$v_{4}$ & 1.42 & 1.76 & 0.87 & -0.65 & 0.28 & 0.492 & 0.902 \\
\hline
\end{tabular}

Fuente: Elaboración propia.

Nota: $\mathrm{ME}=$ Media, $\mathrm{DE}=$ Desviación Estándar, $\alpha=$ alfa de Cronbach, Correlación media entre los ítems $=0.399 \mathrm{y} \alpha$ total $=0.904$. 


\subsection{Validez de constructo y análisis de fiabilidad}

AFE. La factibilidad de la factorización fue pertinente, dado que el $98.3 \%$ de los valores de correlación de la matriz policórica entre los ítems fueron ${ }^{3} 0.31$ (ver Tabla 6). Se obtuvo un alto grado de intercorrelación de las variables (determinante de la matriz de 3.891427e-05, cercano a cero), una adecuación maravillosa del muestreo $(K M O=0.92)$ y una prueba de esfericidad de Bartlett estadísticamente significativa $\left(c^{2}=\right.$ 20,146.32, $g l=105, p<.001)$. La retención de 4 factores fue sugerida por el análisis paralelo basado en la matriz de correlación reducida.

TABLA 6

Matriz de correlaciones

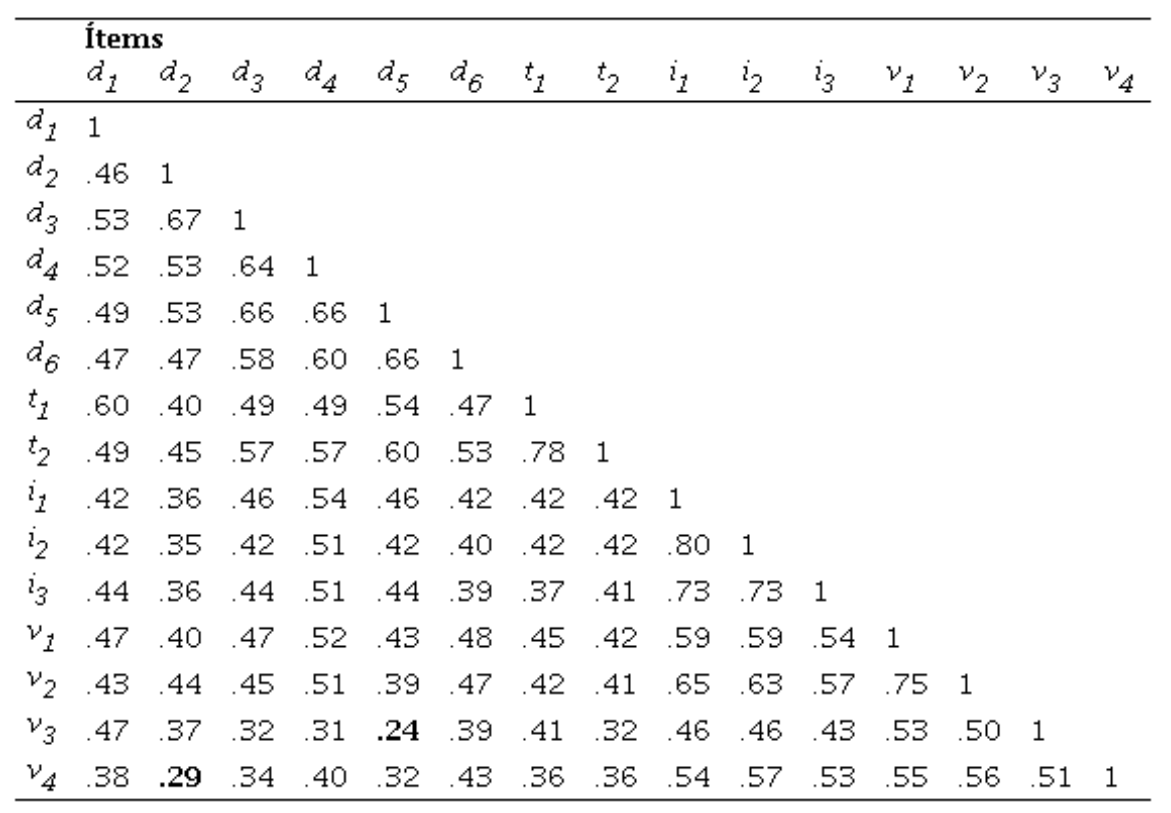

Fuente: Elaboración propia.

Fuente: Elaboración propia.

La Tabla 7 muestra que los 4 factores rotados resultantes tienen asociados los ítems considerados como fuertes, con carga factorial $>0.3$ y comunalidad promedio $>0.5$. El factor rotado 1 fue el de mayor contribución, con una varianza explicada del $23.24 \%$. Entre todos los factores rotados explicaron una varianza del $66.597 \%$, y la fiabilidad fue buena para los 4 factores rotados, debido a los coeficientes $\alpha>0.8$ y $w>0.8$. Los factores resultantes del AFE sustentaron el diseño de las dimensiones planteadas en la versión inicial. 
TABLA 7

Validez del Análisis Factorial Exploratorio

\begin{tabular}{|c|c|c|c|c|c|}
\hline \multirow{2}{*}{ Ítem } & \multicolumn{4}{|c|}{ Componentes rotados } & \multirow{2}{*}{$\begin{array}{l}\text { Comunalidades } \\
\text { Extracción }\end{array}$} \\
\hline & 1 & 2 & 3 & 4 & \\
\hline$d_{3}$ & 0.847 & & & & 0.709 \\
\hline$a_{5}$ & 0.790 & & & & 0.721 \\
\hline$d_{2}$ & 0.707 & & & & 0.513 \\
\hline$a_{4}$ & 0.653 & & & & 0.650 \\
\hline$a_{5}$ & 0.628 & & & & 0.546 \\
\hline$a_{1}$ & 0.343 & & & & 0.492 \\
\hline$i_{2}$ & & 0.895 & & & 0.803 \\
\hline$i_{1}$ & & 0.866 & & & 0.790 \\
\hline$i_{3}$ & & 0.763 & & & 0.667 \\
\hline$t_{1}$ & & & 1.064 & & 1.107 \\
\hline$t_{2}$ & & & 0.514 & & 0.646 \\
\hline$v_{3}$ & & & & 0.586 & 0.521 \\
\hline$v_{1}$ & & & & 0.496 & 0.660 \\
\hline$v_{2}$ & & & & 0.462 & 0.669 \\
\hline$v_{4}$ & & & & 0.377 & 0.495 \\
\hline Autovalor & 3.486 & 3.086 & 1.857 & 1.561 & \\
\hline \% Varianza explicada & 23.238 & 20.576 & 12.380 & 10.403 & \\
\hline \% Varianza explicada acumulada & 23.238 & 43.814 & 56.194 & 66.597 & \\
\hline$\alpha$ & 0.838 & 0.864 & 0.824 & 0.792 & \\
\hline$w$ & 0.847 & 0.874 & 0.825 & 0.801 & \\
\hline
\end{tabular}

Fuente: Elaboración propia.

Nota: $\alpha=$ alfa de Cronbach, $\mathrm{w}=$ omega de McDonald, $\alpha$ total $=0.909 \mathrm{y} \mathrm{w}$ total $=0.914$.

AFC. Se confirmó el modelo propuesto, debido a que los índices de bondad de ajuste fueron aceptables, a saber: $G F I=0.948, N F I=0.948, T L I=0.940, C F I=0.952$ y $A G F I=0.926$ fueron buenos $(>0.90)$, y el $R M S E A(95 \% C I)=0.065(0.062-0.069)$ y $S R M R=0.041$ fueron aceptables $(<0.08)$. La Tabla 8 muestra que las cargas factoriales de los ítems fueron satisfactorias $(>0.5)$, y la fiabilidad fue aceptable para todos los factores $(\alpha y \mathrm{w}>0.7)$. 
TABLA 8

Validez del Análisis Factorial Confirmatorio

\begin{tabular}{|c|c|c|c|c|c|c|}
\hline Factor & Ítem & ME & $\mathrm{DE}$ & Carga factorial & $\alpha$ & $w$ \\
\hline \multirow{6}{*}{ Docencia Factor 1} & $d_{3}$ & 4.13 & 1.12 & 0.750 & \multirow{6}{*}{0.839} & \multirow{6}{*}{0.848} \\
\hline & $a_{4}$ & 3.98 & 1.27 & 0.749 & & \\
\hline & $a_{2}$ & 3.88 & 1.21 & 0.683 & & \\
\hline & $\dot{a}_{5}$ & 4.44 & 1.11 & 0.683 & & \\
\hline & $d_{6}$ & 3.85 & 1.33 & 0.661 & & \\
\hline & $d_{1}$ & 3.00 & 1.58 & 0.640 & & \\
\hline \multirow{3}{*}{ Investigación Factor 2} & $i_{1}$ & 2.20 & 1.77 & 0.866 & \multirow{3}{*}{0.854} & \multirow{3}{*}{0.857} \\
\hline & $i_{2}$ & 1.98 & 1.57 & 0.852 & & \\
\hline & $i_{3}$ & 2.04 & 2.08 & 0.756 & & \\
\hline \multirow{2}{*}{ Tutoría Factor 3} & $t_{2}$ & 3.46 & 1.78 & 0.861 & \multirow{2}{*}{0.822} & \multirow{2}{*}{0.822} \\
\hline & $t_{1}$ & 2.83 & 1.92 & 0.813 & & \\
\hline \multirow{4}{*}{ Vinculación Factor 4} & $v_{2}$ & 1.86 & 1.63 & 0.835 & \multirow{4}{*}{0.780} & \multirow{4}{*}{0.780} \\
\hline & $v_{1}$ & 2.04 & 1.67 & 0.834 & & \\
\hline & $v_{4}$ & 1.43 & 1.79 & 0.563 & & \\
\hline & $v_{3}$ & 1.21 & 1.68 & 0.525 & & \\
\hline Global & $\alpha$ toto & $l=0.9$ & $07 \mathrm{ym}$ & total $=0.921$ & & \\
\hline
\end{tabular}

Fuente: Elaboración propia.

Nota: $\mathrm{ME}=$ Media, $\mathrm{DE}=$ Desviación Estándar, $\alpha=$ alfa de Cronbach y $\mathrm{w}=$ omega de McDonald .

La Figura 1 muestra las correlaciones positivas y estadísticamente significativas entre los factores, que varían desde la más débil (0.55) hasta la más fuerte (0.78). 


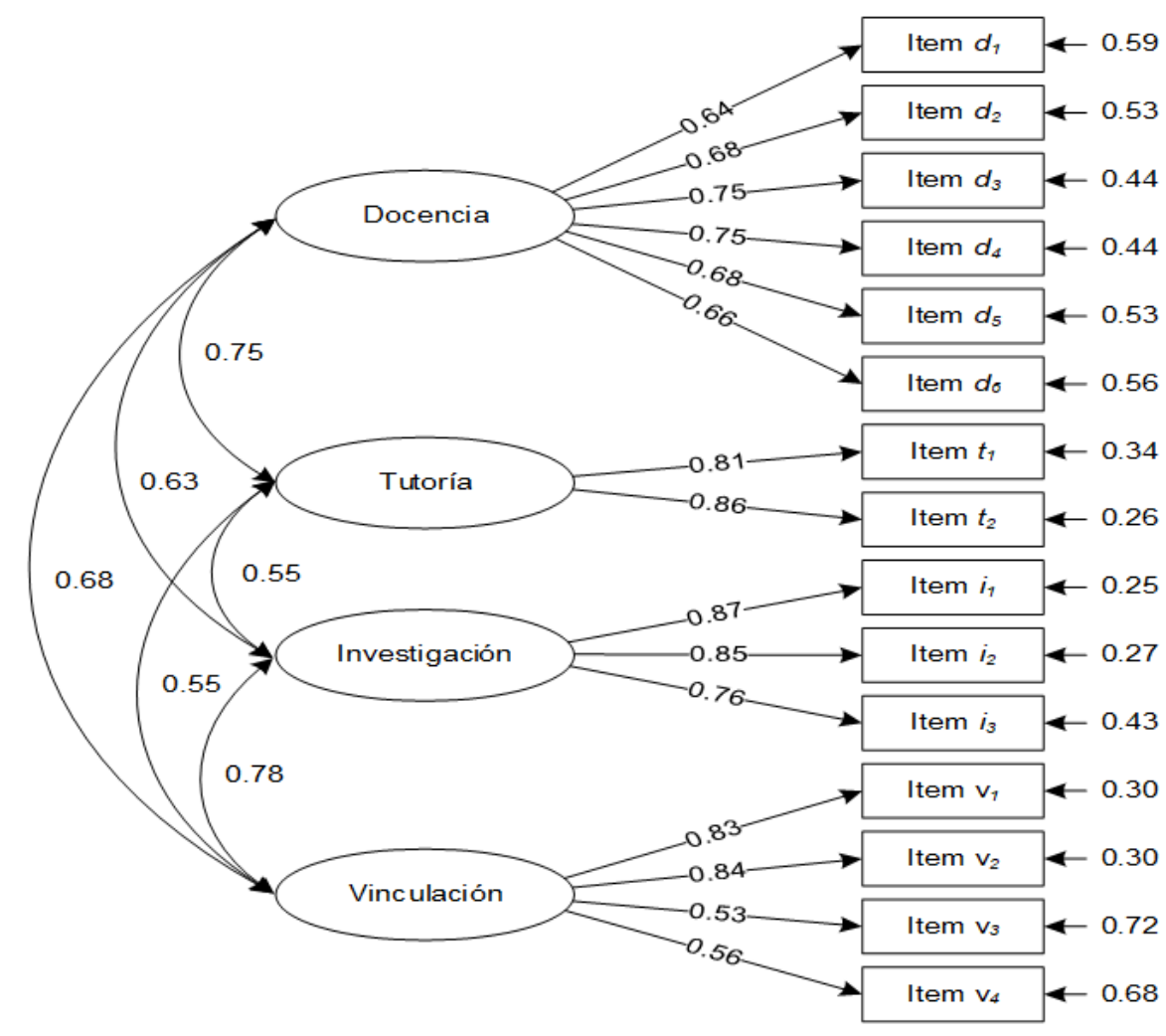

FIGURA 1.

Análisis Factorial Confirmatorio

Fuente: Elaboración propia.

Análisis de fiabilidad. El constructo para evaluar las funciones que desempeña el profesorado, demostró excelente consistencia interna, debido a que los coeficientes totales $\alpha$ y $w$ fueron $>0.9$ (ver Tablas 7 y 8 ).

\subsection{Diagnóstico del desempeño del profesorado}

La Figura 2 muestra la radiografía de la evaluación de las funciones del profesorado del TecNM con respecto a su grado académico y sexo.
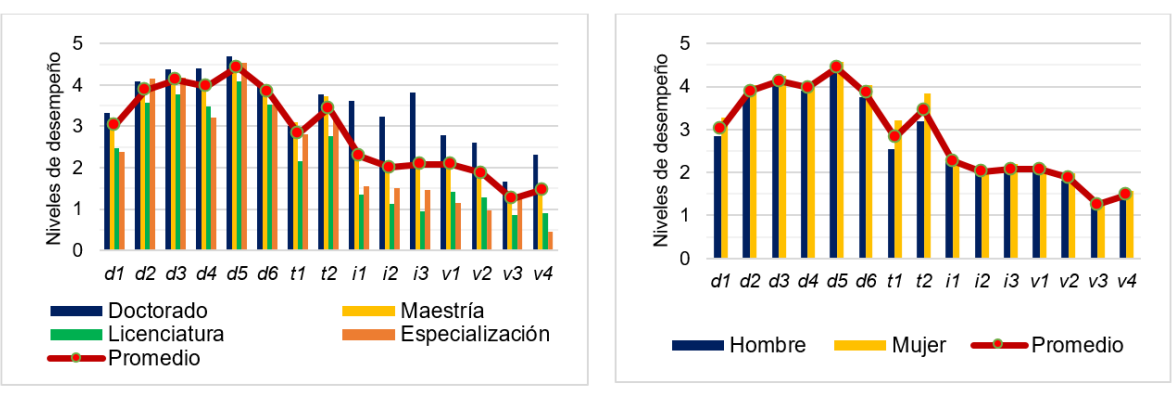

FIGURA 2.

Evaluación de las funciones del profesorado del TecNM con respecto a su grado académico y sexo Fuente: Elaboración propia.

Las gráficas muestran que las dos actividades valoradas muy positivamente están en la dimensión de la docencia; $d_{5}$ indicó que el profesorado en sus clases aplicó recursos educativos con el uso de tecnologías de la 
información e innovó las prácticas en el laboratorio, $\mathrm{y} d$. da evidencia de que el profesorado participó en las reuniones de academia y realizó las actividades académica-administrativas encomendadas. Este último hecho sugiere que el personal docente se preocupó más por las actividades administrativas asignadas que por las actividades dirigidas al aprendizaje del estudiantado. En el otro extremo, las actividades menos valoradas están en la dimensión de vinculación: $v_{2}$ exhibió que el profesorado se involucró escasamente como colaborador en un proyecto multidisciplinar vinculado con el sector empresarial, gubernamental y social, $v_{3}$ probó que el profesorado no impartió cursos de capacitación de formación docente o actualización profesional para el personal de otros campus, y $v$. observó que el profesorado no participó en los comités de evaluación institucional ni tampoco en grupos auditores internos de los sistemas de gestión institucional.

\section{Discusión}

De acuerdo con Maussa (2014) y Cancino y Márquez (2015), se coincide en que el principal propósito de todo sistema de evaluación del profesorado se orienta en tres aspectos: cumplimiento de los fines educativos de la institución, mejoramiento de calidad de la educación y planificación del desarrollo profesional del profesorado. En este sentido, es posible diagnosticar y detectar deficiencias o problemáticas en la institución, donde toma relevancia la evaluación cuando es empleada para elevar la calidad de la educación (Toranzos,1996; Montoya et al., 2014). Por tal motivo, es importante la integración de estrategias de evaluación en las instituciones de educación superior, que obtengan información válida y confiable desde la perspectiva de las diferentes partes que intervienen en el proceso educativo.

Por lo anterior, el instrumento creado en este trabajo pretende aportar desde la perspectiva de las jefaturas de departamento académico, de los 254 campus del TecNM, indicadores que valoren el actuar del profesorado dentro de los espacios educativos de la institución, para identificar fortalezas y debilidades de la planta docente. Las dimensiones de docencia, tutoría, investigación y vinculación, consideradas para valorar las funciones del personal docente, son fundamentales en el quehacer educativo de la institución, tanto al interior como al exterior (Torra et al., 2012). Los ítems de las dimensiones fueron rigurosamente elaborados acorde a todos los procesos académico-administrativos donde participa el profesorado. Las evidencias presentadas de validez de contenido, de comprensión y de constructo del instrumento, en el contexto del TecNM, refieren a un modelo sustentable que permite evaluar las funciones que desempeña el profesorado en educación superior tecnológica, percibida por las jefaturas de departamento académico.

Desde la perspectiva del presente trabajo, las cuatro dimensiones planteadas definen una aproximación del perfil que se espera del profesorado del TecNM, la docencia se refiere a la superación académica, planeación y recursos didácticos de asignatura, asesoría que brinda al estudiantado, la participación en las academias y procesos institucionales (Tejedor, 2003; Perrenoud, 2004; Zabalza, 2007; López et al., 2014); la tutoría considera la acción tutorial que se desarrolla y la capacitación en el tema (Comellas, 2002); la investigación incluye el reconocimiento de los logros investigativos y la participación en redes académicas de generación de conocimiento científico-tecnológico (Rueda et al., 2010); y la vinculación se refiere a la participación en los servicios institucionales que se ofrecen al entorno socioeconómico, como la capacitación, asesoría, desarrollo de proyectos y la asistencia a órganos colegiados (Ruiz-Corbella y Aguilar-Feijoo, 2017).

Los resultados sugieren que el TecNM tiene una cultura de evaluación consolidada, debido a que no se presentaron inconvenientes para la aplicación de la evaluación. La docencia sigue siendo la dimensión más privilegiada y considerada como la principal actividad que realiza el profesorado; se restringe la dimensión de vinculación, lo cual coincide con los resultados presentados por Ruiz-Corbella y Aguilar-Feijoo (2017), en el que la vinculación tuvo una confiabilidad por debajo de un umbral aceptable $\alpha<$.7. Respecto a la formación académica del profesorado, se detectaron diferencias en las dimensiones de investigación y vinculación, entre los que ostentan un grado académico de doctorado comparado con los de maestría, especialización y 
licenciatura, probablemente esto se debe a que se reconocen formaciones más especiales en los roles científicos que se adquieren en el nivel educativo de doctorado (Rueda et al., 2010).

Si bien existen sistemas de evaluación docente que miden las competencias del profesorado desde la opinión del estudiantado (Zambrano et al., 2005; Pimienta, 2014; Luna y Reyes, 2015; Dios et al., 2018) o desde el punto de vista del profesorado (Hoi et al., 2017; Aguiar et al., 2018), siempre es indispensable complementar la evaluación desde de la perspectiva de otro actor en la institución: la autoridad educativa. Además, los diversos instrumentos que se han desarrollado no siempre presentan evidencias de validez y confiabilidad, lo cual limita comprobar el modelo generado.

El instrumento propuesto demostró ser una herramienta para el TecNM en la medición del nivel del quehacer docente institucional; sin embargo, existen ciertas limitaciones: la primera, que no es posible generalizar los resultados a la educación superior en México debido a que los hallazgos encontrados están relacionados con las características de los 254 institutos tecnológicos del TecNM; la segunda, que es recomendable incrementar el número de personas jueces e incluir participantes de otras instituciones que aporten otras experiencias y perspectivas. Para futuras investigaciones se espera complementar la evaluación con la integración de instrumentos de la opinión del estudiantado (clases, titulación, estancias, servicio social y tutoría) y otro de atención a las personas con necesidades específicas educativas (enfoque incluyente).

\section{Conclusiones y RECOMENDACIONES}

Los resultados y evidencia empírica aportan un estudio a la literatura de la práctica de la evaluación del profesorado de educación superior tecnológica, donde se considera el contexto del TecNM desde la perspectiva de la autoridad.

Se identificó un perfil para el profesorado, el cual resulta coherente con la actuación de la institución: docencia, tutoría, investigación y vinculación. La dimensión de la docencia fue la más valorada positivamente, y la de vinculación, la menos valorada; se encontraron diferencias en esta última según el grado académico. El profesorado que ostenta doctorado exhibe una valoración superior al promedio, mientras que el complemento exhibe una valoración menor. El instrumento de 15 ítems desarrollado mostró un proceso de rigurosa revisión por un jurado experto y una alta consistencia interna, por lo que se puede considerar como una escala útil para valorar las funciones de docencia, tutoría, investigación y vinculación. Se obtuvo una radiografía del estado que guarda el perfil del profesorado, al identificar las fortalezas y debilidades en las cuatro dimensiones.

A partir de los resultados, y según el contexto del TecNM, se sugieren las siguientes recomendaciones:

- Utilizar las tecnologías de la información para que el sistema de evaluación sea de fácil acceso para todas las personas involucradas en el proceso, particularmente en el profesorado, para la interpretación de los resultados y retroalimentación de su práctica docente.

- Implementar de forma sistémica y con estatus de institucional la evaluación del profesorado, en el sentido de reflexión de la práctica educativa en una institución, y evitar que sea de control en las relaciones laborales.

- Reconocer la evaluación del profesorado como una herramienta institucional útil para la toma de decisiones en la capacitación y desarrollo profesional, sustentado a partir de las debilidades y fortalezas detectadas en el proceso.

- Utilizar más instrumentos acordes al perfil del profesorado, que evalúen la percepción del estudiantado, personal directivo y personas familiares. El diseño de los instrumentos debe responder al contexto institucional. 


\section{Agradecimientos}

Esta investigación ha sido posible gracias a la colaboración del Tecnológico Nacional de México a través de la Dirección de Docencia e Innovación Educativa, quien apoyó en la recopilación de la información, y de los profesores José Ángel Jesús, Ezequiel Gómez y José Manuel Gómez del Instituto Tecnológico de Villahermosa, quienes ayudaron en la programación de la plataforma informática.

\section{REFERENCIAS BIBLIOGRÁFICAS}

Aguiar, M. E., Gutiérrez, H. y Gutiérrez, P. (2018). Diseño y aplicación de un cuestionario sobre la práctica docente del profesorado de matemáticas en ingeniería y ciencias. Góndola, Enseña y Aprendizaje de las Ciencias, 13(1), 33-54. doi: https://doi.org/10.14483/23464712.11732

Arreola, R. L. (2019). Formación y evaluación docente basada en un perfil por competencias. Una propuesta desde la práctica reflexiva. Revista Educación, 43(2), 2215-2644. doi: https://doi.org/10.15517/revedu.v43i2.30898

Ayala, S. y O'Hinggins, T. (2019). Perfil docente de la educación superior inclusiva en Paraguay. Revista Cientifica en Ciencias Sociales, 1(1), 75-84. Recuperado de https://bit.ly/3pd0qUy

Cancino, E. y Márquez, S. (2015). Evaluación de desempeño de la función académica: análisis de un sistema en el contexto universitario chileno. Formación universitaria, 8(3), 35-46. doi: https://doi.org/10.4067/S0718-500 62015000300005

Comellas, M. J. (2002). Las competencias del profesorado para la acción tutorial. Bilbao, España: Praxis.

Cruz-Aguayo, Y., Hincapié, D. y Rodríguez, C. (2020). Profesores a prueba. Claves para una evaluación docente exitosa. Washington D. C., Estados Unidos: Banco Interamericano de Desarrollo.

Dios, I., Calmaestra, J. y Rodríguez-Hidalgo, A. J. (2018). Validación de la escala de competencias docentes organizacionales y didácticas para educadores. Revista Mexicana de Investigación Educativa, 23(76), 281-302. Recuperado de https://bit.ly/36rLzgk

Dirección General de Educación Superior Tecnológica [DGEST]. (2006). Manual de los procesos de evaluación del desempeño docente y del ingreso de aspirantes a la educación superior tecnológica 2006-2007. Distrito Federal, México: Coordinación Editorial del Instituto Tecnológico de Durango.

Fernández, J. (2013). Competencias docentes y educación inclusiva. Revista Electrónica de Investigación Educativa, 15(2), 82-99. Recuperado de https://bit.ly/3eJG2We

Gómez, L. F. y Valdés, M. G. (2019). La evaluación del desempeño docente en la educación superior. Propósitos y Representaciones, 7(2), 479-515. doi: https://doi.org/10.20511/pyr2019.v7n2.255

Hoi, C., Zhou, M., Teo, T. y Nie, Y. (2017). Measuring efficacy sources: Development and validation of the sources of teacher efficacy questionnaire (STEQ) for chinese teachers [Medición de fuentes de eficacia: desarrollo y validación de las fuentes del cuestionario de eficacia docente (STEQ) para maestros chinos]. Psychology in the Schools, 54(7), 756-769. doi: https://doi.org/10.1002/pits.22025

Isoré, M. (2009). Teacher evaluation: current practices in OECD countries and a literature review [Evaluación del profesorado: prácticas actuales en los países de la OCDE y una revisión de la literatura]. OECD Education Working Papers, (23), 1-48. doi: https://doi.org/10.1787/223283631428

López, A. B., González, I. y de León, C. (2014). Perfil de un buen docente. Aplicación de un protocolo de evaluación de las competencias del profesorado universitario. Revista Electrónica Interuniversitaria de Formación del Profesorado, 17(1), 133-148. doi: https://doi.org/10.6018/reifop.17.1.190531

Luna, E. y Reyes, E. P. (2015). Validación de constructo de un cuestionario de evaluación de la competencia docente. Revista Electrónica de Investigación Educativa, 17(3), 13-27. Recuperado de https://bit.ly/36rpTkq

Maussa, E. J. (2014). Características, principios y fines de la evaluación del desempeño docente. Revista Praxis, 10(1), 8-20. doi: https://doi.org/10.21676/23897856.1355 
Arturo Carranza, et al. Evaluación de las funciones que desempeña el profesorado en educación Supe...

Mohamadi, Z. y Malekshahi, N. (2018). Designing and validating a potential formative evaluation inventory for teacher competences [Diseñando y validando un potencial inventario de evaluación formativa de las competencias docentes]. Lang Test Asia, 8(6), 1-21. doi: https://doi.org/10.1186/s40468-018-0059-2

Montoya, J., Arbesú, I., Contreras, G. y Conzuelo, S. (2014). Evaluación de la docencia universitaria en México, Chile y Colombia: Análisis de experiencias. Revista Iberoamericana de Evaluación Educativa, 7(2e), 15-42. Recuperado de http://bit.ly/37AG9lb

Moreno, M., Nava, M. y Campos, M. (2014). Los comentarios abiertos como referente de evaluación de la docencia universitaria: la conveniencia de su interpretación y tratamiento. Formación universitaria, 7(1), 41-48. doi: htt ps://doi.org/10.4067/S0718-50062014000100006

Pacheco, M., Ibarra, I., Iñiguez, M. L., Lee, H. y Sánchez, V. (2018). La evaluación del desempeño docente en la educación superior. Revista Digital Universitaria (RDU), 19(6), 1-11. doi: https://doi.org/10.22201/codeic.1 6076079e.2018.v19n6.a2

Perrenoud, P. (2004). Diez nuevas competencias para enseñar. Barcelona, España: Editorial GRAO.

Pimienta, J. (2014). Elaboración y validación de un instrumento para la medición del desempeño docente basado en competencias. REDU. Revista de Docencia Universitaria, 12(2), 231-250. doi: https://doi.org/10.4995/redu.2 014.5648

Rueda, M., Luna, E., García, B. y Loredo, J. (2010). La evaluación de la docencia en las universidades públicas mexicanas: un diagnóstico para su comprensión y mejora. Revista Iberoamericana de Evaluación Educativa, 3(1e), 77-92. Recuperado de https://bit.ly/2Irllnr

Ruiz-Corbella, M. y Aguilar-Feijoo, R. M. (2017). Competencias del profesor universitario: elaboración y validación de un cuestionario de autoevaluación. Revista Iberoamericana de Educación Superior, 8(21), 37-65. doi: https:/ /doi.org/10.22201/iisue.20072872e.2017.21.212

Sanders, W. L., Wright, S. P. y Horn, S. P. (1997). Teacher and Classroom Context Effects on Student Achievement: Implications for Teacher Evaluation [Efectos del contexto del profesorado y del aula en el rendimiento de los estudiantes: Implicaciones para la evaluación del profesorado].Journal of Personnel Evaluation in Education, 11, 57-67 doi: https://doi.org/10.1023/A:1007999204543

Secretaría de Educación Pública [SEP]. (1982, 21 de abril). Título séptimo. Procedimientos para el ingreso y promoción del personal docente. En Reglamento interior de trabajo del personal docente de los Institutos Tecnológicos. SEP. Recuperado de https://bit.ly/37EyQJ9

Tejedor, F. (2003). Un modelo de evaluación del profesorado universitario. Revista de Investigación Educativa, 21(1),157-182. Recuperado de https://bit.ly/3eJLNmI

Tejedor, F. y Jornet, J. M. (2008). La evaluación del profesorado universitario en España. Revista Electrónica de Investigación Educativa, 10, 1-29. Recuperado de http://bit.ly/2LNHQmF

Toranzos, L. (1996). Evaluación y calidad. Revista Iberoamericana de Educación, 10, 63-78. doi: https://doi.org/10. $35362 /$ rie 1001167

Torra, I., de Corral, I., Pérez, M. J., Triadó, X., Pagès, T., Valderrama, E., ... y Tena, A. (2012). Identificación de competencias docentes que orienten el desarrollo de planes de formación dirigidos a profesorado universitario. REDU. Revista de Docencia Universitaria, 10(2), 21-56. doi: https://doi.org/10.4995/redu.2012.6096

Zabalza, M. (2007). Competencias docentes del profesorado universitario. Calidad y desarrollo profesional (2a ed.). Madrid, España: Narcea.

Zambrano, R., Meda, R. y Lara, B. (2005). Evaluación de profesores universitarios por parte de los alumnos mediante el Cuestionario de Evaluación de Desempeño Docente (CEDED). Revista de Educación y Desarrollo, 4, 63-69. Recuperado de https://bit.ly/38pDdZo

\section{INFORMACIÓN ADICIONAL}


Cómo citar: Gamino Carranza, A., Castro Ramírez, S.L. y García Domínguez, M. (2021). Evaluación de las funciones que desempeña el profesorado en educación superior tecnológica. Revista Educación, 45(2). Recuperado de http://doi.org/10.15517/revedu.v45i1.44372 